\title{
A SPACE OF SMALL SPREAD WITHOUT THE USUAL PROPERTIES
}

\author{
JUDITH ROITMAN
}

\begin{abstract}
A space is found, for any $\alpha$, which has spread $\alpha$ and which is not the set-theoretic union of a hereditarily $\alpha$-Lindelof and a hereditarily $\alpha$-separable space.
\end{abstract}

Introduction. At the 1972 Bolyai János Mathematical Society Colloquium, A. Hajnal and I. Juhasz noted that every known Hausdorff space of spread $\omega$ was the union of a hereditary separable space and a hereditarily Lindelof space. The main result of this paper is a family of counterexamples to a generalization of this situation; the method of proof will also yield, in Lemma 2(c), a family of spaces such that no "large" subspaces are regular.

Some notational conventions. If $X$ is a space, by its topology $\mathscr{T}$ we mean the family of open sets; if $\mathscr{A}$ is a family of subsets of $X$, the topology on $X$ induced by $\mathscr{T} \cup \mathscr{A}$ is the closure of $\mathscr{T} \cup \mathscr{A}$ under arbitrary union and finite intersection. We write $\langle X, \mathscr{T}\rangle$ for $X$ with the topology $\mathscr{T}$; if $Y \subset X,\langle Y, \mathscr{T}\rangle$ means $\langle Y,\{u \cap Y: u \in \mathscr{T}\}\rangle$. Given any set $S,|S|$ denotes the cardinality of $S$.

\section{Statement of results.}

Definition. Given a topological space $X$, we define its spread by

$$
\operatorname{sp}(X)=\sup \{|Y|: Y \text { is a discrete subspace of } X\} .
$$

Definition. Let $\alpha$ be any cardinal, $X$ a space. Then $X$ is $\alpha$-Lindelof iff every open cover of $X$ has a subcover of cardinality $\leqq \alpha$. Similarly, $X$ is $\alpha$-separable iff every subspace has a dense set of cardinality $\leqq \alpha$.

Definition. Let $X$ be a space, $P$ any property of topological spaces. Then $X$ is hereditarily $P$ iff every subspace of $X$ has property $P$.

We note that if $X$ is either hereditarily $\alpha$-separable or hereditarily $\alpha$-Lindelof, $\operatorname{sp}(X) \leqq \alpha$.

Received by the editors June 12, 1973.

AMS (MOS) subject classifications (1970). Primary 54C15, 54G20; Secondary 54D99, 54D20, 54D10.

Key words and phrases. Spread, Lindelof, separable. 
THEOREM. Let $\alpha$ be a cardinal. Then there exists a Hausdorff space $X$ of cardinality $\alpha^{+}$such that $\operatorname{sp}(X)=\alpha$ and $X$ is not the set-theoretic union of a hereditarily $\alpha$-Lindelof space and a hereditarily $\alpha$-separable space.

COROLlARY OF PROOF. For every cardinal $\alpha$ there exists a Hausdorff space of cardinality $\alpha^{+}$with no regular subspaces of cardinality $\alpha^{+}$.

Construction. From now on we fix some cardinal $\alpha$. The construction proceeds by taking a space $X$ of cardinality $\alpha^{+}$which is hereditarily $\alpha$-separable and hereditarily $\alpha$-Lindelof (any $X \subseteq 2^{\alpha},|X|=\alpha^{+}$will do). The points are then thought of as being indexed by the "square" array $\alpha^{+} \times \alpha^{+}$. Lemma 1 ensures that no "vertical" or "diagonal" section is Lindelof; Lemma 2 ensures that no "horizontal" section is separable.

Lemma 1. Let $X$ be a hereditarily $\alpha$-separable space under the topology $\mathscr{T}$, and suppose $X$ is the disjoint union of $\alpha^{+}$nonempty sets, $X=\bigcup_{\beta<\alpha^{+}} X_{\beta}$. Let $\mathscr{T}^{\prime}$ be the topology induced on $X$ by $\mathscr{T} \cup\left\{\bigcup_{\beta \leqq \gamma} X_{\beta}: \gamma<\alpha^{+}\right\}$. Then

(a) $\left\langle X, \mathscr{T}^{\prime}\right\rangle$ is not $\alpha$-Lindelof; in fact if $Y \subseteq X,\left|\left\{\beta: Y \cap X_{\beta} \neq \varnothing\right\}\right|=\alpha^{+}$ then $Y$ is not $\alpha$-Lindelof.

(b) $\left\langle X_{\beta}, \mathscr{T}^{\prime}\right\rangle=\left\langle X_{\beta}, \mathscr{T}\right\rangle$ for all $\beta\left\langle\alpha^{+}\right.$. Thus if $X$ is hereditarily $\alpha-$ Lindelof under $\mathscr{T},\left\langle X_{\beta}, \mathscr{T}^{\prime}\right\rangle$ will be both hereditarily $\alpha$-Lindelof and hereditarily $\alpha$-separable.

(c) $\left\langle X, \mathscr{T}^{\prime}\right\rangle$ is hereditarily $\alpha$-separable.

Proof. (a) Let $Y$ be as in the hypothesis, and consider the open cover of $Y,\left\{Y \cap \bigcup_{\beta \leqq \gamma} X_{\beta}: \gamma<\alpha^{+}\right\}$. Clearly no subfamily of cardinality $\alpha$ will cover $Y$.

(b) Clear.

(c) Let $Y \subseteq X$. Let $A$ be a dense set of cardinality $\leqq \alpha$ for $\langle Y, \mathscr{T}\rangle$, and let $\gamma=\sup \left\{\beta: A \cap X_{\beta} \neq \varnothing\right\}$. If $y \in Y \cap \bigcup_{\beta \geq \gamma} X_{\beta}$ and $y \in u \in \mathscr{T}^{\prime}$ then $u \cap A \neq \varnothing$. For $\beta \leqq \gamma$, let $A_{\beta}$ be dense for $\left\langle Y \cap X_{\beta}, \mathscr{T}^{\prime}\right\rangle,\left|A_{\beta}\right| \leqq \alpha$. Then $A \cup \bigcup_{\beta \leqq \gamma} A_{\beta}$ is dense for $\left\langle Y, \mathscr{T}^{\prime}\right\rangle$ and has cardinality $\leqq \alpha$.

Lemma 2. Let $X=\left\{x_{\beta}: \beta<\alpha^{+}\right\}$be a hereditarily $\alpha$-Lindelof space of cardinality $\alpha^{+}$with topology $\mathscr{T}$. Let $\mathscr{A}$ be any collection of subsets of $X$ such that $|X-A| \leqq \alpha$ for all $A \in \mathscr{A}$. Let $\mathscr{T}^{\prime}$ be the topology induced on $X$ by $\mathscr{T} \cup \mathscr{A}$. Then

(a) $\left\langle X, \mathscr{T}^{\prime}\right\rangle$ is hereditarily $\alpha$-Lindelof.

(b) If, for all $\gamma\left\langle\alpha^{+},\left\{x_{\beta}: \beta \geqq \gamma\right\} \in \mathscr{A}\right.$, then $\left\langle X, \mathscr{T}^{\prime}\right\rangle$ is not $\alpha$-separable.

(c) If, for all $\gamma\left\langle\alpha^{+},\left\{x_{\beta}: \beta \geqq \gamma\right\} \in \mathscr{A}\right.$ and $\langle X, \mathscr{T}\rangle$ is hereditarily $\alpha$ separable, then $\forall Y \subseteq X\left(|Y|=\alpha^{+} \rightarrow\left\langle Y, \mathscr{T}^{\prime}\right\rangle\right.$ is not regular $)$.

Proof. (a) Let $Y \subseteq X, B \subset \mathscr{T}^{\prime}$ be a basic open cover of $Y$. We may assume $\mathscr{A}$ is closed under finite intersection. Then $\forall b \in B, b=u \cap v$ for some $u \in \mathscr{T}, v \in \mathscr{A}$. Let $\mathscr{B}_{\mathscr{T}}=\{u \in \mathscr{T}: \exists b \in \mathscr{B}, \exists v \in \mathscr{A}(b=u \cap v)\}$, 
and let $\mathscr{C} \subseteq \mathscr{B}_{\mathscr{T}}$ be a subcover of $Y,|\mathscr{C}| \leqq \alpha$. Then $\forall u \in \mathscr{C}, \exists b \in \mathscr{B}$ such that $|u-b| \leqq \alpha$. For each $u \in \mathscr{C}$, fix such a $b \in \mathscr{B}$, calling it $b_{u}$, and let $\mathscr{C}_{u} \subset \mathscr{T}^{\prime}$ cover $\left(u-b_{u}\right) \cap Y,\left|\mathscr{C}_{u}\right| \leqq \alpha$. Then $\left\{b_{u}: u \in \mathscr{B}_{\mathscr{T}}\right\} \cup \bigcup_{u \in \mathscr{B}_{\mathscr{I}}} \mathscr{C}_{u}$ is a subcover of $Y$ in $\mathscr{T}^{\prime}$ of cardinality $\alpha$.

(b) Let $A \subseteq X,|A| \leqq \alpha$. Let $\gamma=\sup \left\{\beta: x_{\beta} \in A\right\}$. Then $\left\{x_{\delta}: \delta>\gamma\right\}$ is open and $A \cap\left\{x_{\delta}: \delta>\gamma\right\}=\varnothing$.

(c) Let $Y \subseteq X,|Y|=\alpha^{+}$. Since $\left\langle X, \mathscr{T}^{\prime}\right\rangle$ is hereditarily $\alpha$-Lindelof, we may without loss of generalization, assume that all open sets of $\left\langle Y, \mathscr{T}^{\prime}\right\rangle$ have cardinality $\alpha^{+}$. Suppose $A$ is dense in $\langle Y, \mathscr{T}\rangle,|A| \leqq \alpha$. Again, let $\gamma=\sup \left\{\delta: x_{\delta} \in A\right\}$. Suppose $\beta>\alpha$. Then $x_{\beta}$ is not an element of the closed set $\left\{x_{\delta}: \delta \leqq \gamma\right\}=w_{\gamma}$. We show that $x_{\beta}$ and $w_{\gamma}$ cannot be separated by open sets in $\mathscr{T}^{\prime}$.

Let $u, v \in \mathscr{T}^{\prime}, x_{\beta} \in u, w_{\gamma} \subset v$. Then $u=u^{\prime} \cap a, v=v^{\prime} \cap c$ for some $u^{\prime}$, $v^{\prime} \in \mathscr{T}$, and $a, c \in \mathscr{A}$. Since $A$ is dense relative to $\mathscr{T}, u^{\prime} \cap v^{\prime} \neq \varnothing$; hence $\left|u^{\prime} \cap v^{\prime}\right|=\alpha^{+}$. But then $|u \cap v|=\left|u^{\prime} \cap v^{\prime} \cap a \cap c\right|=\alpha^{+}$; clearly $u \cap v \neq \varnothing$.

Proposition. There exists a Hausdorff space $X$ of spread $\alpha$ such that if $X=Y_{0} \cup Y_{1}$ then $\exists i \exists Z \exists Z^{\prime}\left(Z \subseteq Y_{i}, Z^{\prime} \subseteq Y_{i}, Z\right.$ is not $\alpha$-separable and $Z^{\prime}$ is not $\alpha$-Lindelof).

Proof. Let $X$ be a hereditarily $\alpha$-separable, hereditarily $\alpha$-Lindelof Hausdorff space of spread $\alpha, X=\bigcup_{\beta<\alpha^{+}} X_{\beta}$ as in Lemma 1, and suppose each $X_{\beta}$ has cardinality $\alpha^{+}$. Let $\mathscr{T}^{\prime}$ be as in Lemma 1 . We list the elements of $X_{\beta}$ as $\left\{X_{\delta}^{\beta}: \delta<\alpha^{+}\right\}$and note that $\left\langle X_{\beta}, \mathscr{T}^{\prime}\right\rangle$ is hereditarily $\alpha$-separable and hereditarily $\alpha$-Lindelof. Let $\mathscr{A}_{\beta}$ be as in Lemma 2 (b) for $X_{\beta}$. We construct the topology $\mathscr{T}^{*}$ as follows:

Given $x_{\delta}^{\beta} \in X, u \in \mathscr{T}^{\prime}, v \in \mathscr{A}_{\beta}$ such that $x_{\delta}^{\beta} \in u \cap v$, the following is a neighborhood basic open set: $u \cap\left[v \cup \bigcup_{\rho<\beta} X_{\rho}\right]$.

These sets are closed under finite intersection, hence they form a basis. Let $\mathscr{T}^{*}$ be the topology they generate. Clearly $\left\langle X, \mathscr{T}^{*}\right\rangle$ is Hausdorff and has spread $\geqq \alpha$. We show the spread is $\alpha$ : Suppose $Y \subseteq X,|Y|=\alpha^{+}$. Then either

(a) $\exists Z \subseteq Y$ such that $\left|\left\{\beta: Z \cap X_{\beta} \neq \varnothing\right\}\right|=\alpha^{+}$, or

(b) $\exists Z \subseteq Y$ such that $|Z|=\alpha^{+}$and for some $\beta<\alpha^{+}, Z \subseteq X_{\beta}$.

In case (a) we may assume $\left|Z \cap X_{\beta}\right| \leqq 1$ for all $\beta\left\langle\alpha^{+}\right.$. Then $\left\langle Z, \mathscr{T}^{*}\right\rangle=$ $\left\langle Z, \mathscr{T}^{\prime}\right\rangle$ and by Lemma $1, Z$ is hereditarily $\alpha$-separable, hence not discrete. In case (b), by Lemma $2, Z$ is hereditarily $\alpha$-Lindelof, hence not discrete. In either case, $Y$ is not discrete. Now suppose $X=Y_{0} \cup Y_{1}$. Suppose $\left|\left\{\beta: Y_{0} \cap X_{\beta} \neq \varnothing\right\}\right|<\alpha^{+}$. Then letting $\gamma=\sup \left\{\beta: Y_{0} \cap X_{\beta} \neq \varnothing\right\}$ we have $Y_{1} \cap X_{\gamma+1}$ which is not $\alpha$-separable, and $\left\{x_{0}^{\delta}: \delta>\gamma\right\}$ is a non- $\alpha$-Lindelof subspace of $Y_{1}$. So we can assume $\left|\left\{\beta: Y_{i} \cap X_{\beta} \neq \varnothing\right\}\right|=\alpha^{+}$for each $i$. 
Hence neither $Y_{0}$ not $Y_{1}$ is $\alpha$-Lindelof. Consider some $\delta<\alpha^{+}$. Then $\left|X_{\delta} \cap Y_{i_{0}}\right|=\alpha^{+}$for some $i_{0}$. But then $X_{\delta} \cap Y_{i_{0}}$ is not $\alpha$-separable, and this completes the proof.

In closing, we notice that by Lemma 2(c) this space is most definitely not regular; it would be interesting to know if a regular space can satisfy the main theorem.

\section{REFERENCES}

1. A. Hajnal and I. Juhasz, On hereditarily $\alpha$-Lindelöf and $\alpha$-separable spaces, Ann. Univ. Sci. Budapest Eötvös Sect. Math. 11 (1968), 115-124. MR 39 \#2124.

2. - A consistency result concerning hereditarily $\alpha$-separable spaces, Proceedings of the Bolyai János Mathematical Society Colloquium on Topology, Keszthely, Hungary, 1972 (to appear).

Department of Mathematics, University of California, Berkeley, California 94720 